\title{
Ampullary Crest
}

National Cancer Institute

\section{Source}

National Cancer Institute. Ampullary Crest. NCI Thesaurus. Code C32063.

A sensory receptor of the ear located in the membranous ampulla of the semicircular duct. 